\title{
Recursive Bayesian Estimation for Respiratory Motion Correction in Nuclear Medicine
}

\author{
Rhodri L Smith, Ashrani Abd Rahni, John Jones, and Kevin Wells,
}

Acess online http://ieeexplore.ieee.org/stamp/stamp.jsp?arnumber=06551672

\begin{abstract}
Respiratory motion correction degrades quantitatively and qualitatively Nuclear Medicine images. We propose that adaptive approaches are required to correct for the irregular breathing patterns often encountered in the clinical setting, which can be addressed within a Bayesian tracking formulation. This allows inference of the hidden organ configurations using only knowledge of an external observation such as a parametrized external surface. The flexible framework described provides a method to correct for organ motion whilst accommodating for irregular unseen respiratory patterns. In this work we utilize a Kalman filter and compare it with a Particle filter. A novel adaptive state transition model is also introduced to describe the evolution of organ configurations. The Kalman filter marginally outperforms the Particle filter, both approaches however offer an effective motion correction mechanism, correcting for motion with errors of around $1-3 \mathrm{~mm}$. We present results of simulated PET images derived from XCAT to demonstrate the efficacy of the approach.
\end{abstract}

Index Terms-Respiratory motion correction, adaptive, recursive Bayesian estimation.

\section{INTRODUCTION}

$\mathbf{N}$ UCLEAR Medicine (NM) Imaging is a powerful technique in visualizing radio-pharmaceutically targeted physiological processes with wide ranging clinical applications in oncology, cardiology and neurology. Increasing performance in both reconstruction algorithms and detector technology in recent years has motivated the need for the correction of respiratory motion artefacts. Numerous approaches to correct for motion have been proposed [1] [2] [3]; many of which rely on regular respiratory motion to be effective. More advanced approaches utilize patient specific motion models but do not account for the variability in the patients breathing pattern [4]. However by considering the problem in a Bayesian filtering context [5] allows the implementation of an adaptive framework to address this issue.

In this new work we investigate this adaptive tracking approach, within the Bayesian estimation paradigm: we use a Kalman filter (KF) and compare this with a Particle filter (PF) [6]. The respiratory correction framework proposes to infer the hidden geometrical internal organ deformations, $x_{k}$, from an observed stereo surface capture of the anterior portion of the external surface of the torso, $z_{k}$. Describing the framework as a Hidden Markov Model (HMM) allows inference of the "true" organ deformation given the previously unseen observations (figure 1).

Presented 29/10 - 11/3, 2012, Disneyland Hotel, Anaheim, California, doi: 10.1109/NSSMIC.2012.6551672 any questions contact R. Smith (email:rhodri.smith@surrey.ac.uk)

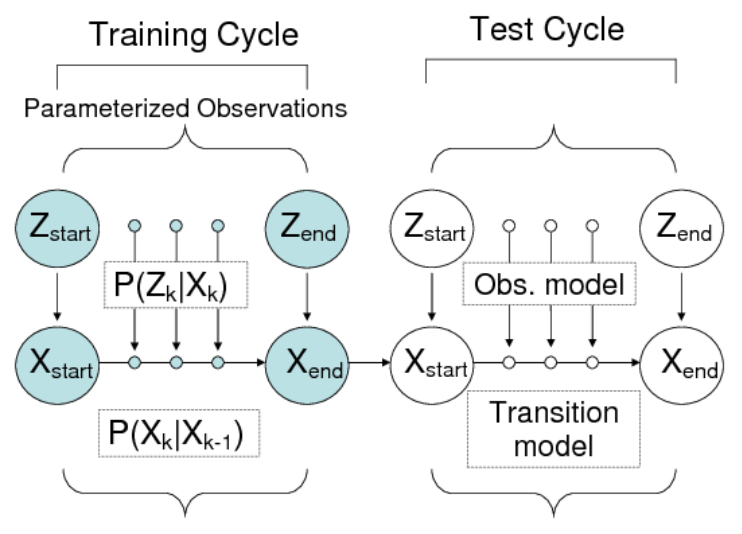

Registration parameters from Inferred registration parameters Multi frame low dose CT

Fig. 1. Schematic diagram motion correction framework

\section{METHOD}

Bayesian filtering describes the abstract probabilistic framework to sequentially infer a hidden state $x_{k}$ given a series of observations $z_{1: k}$. The state of the internal organs can thus be considered as a stochastic process with a Markov property. A transition model is employed to describe the evolution of organ configurations over a discrete time index $k$. Each hidden state is related to an observation through an observation model, wherein each observation is mutually independent given the hidden state. The state transition and observation can be described in their generic probabilistic state space form (equation 1 ).

$$
x_{k} \sim p\left(x_{k} \mid x_{k-1}\right) \quad z_{k} \sim p\left(z_{k} \mid x_{k}\right)
$$

Inference of the hidden state can be described in a predictupdate cycle. Given an initial state $p\left(x_{k-1} \mid x_{1: k-1}\right)$ of a dynamical system; a prediction of the state is made $p\left(\boldsymbol{x}_{\boldsymbol{k}} \mid z_{1: k-1}\right)$ via the transition model. The update phase then incorporates the current observed information $z_{k}$ to refine the state estimate, resulting in the a posteriori state estimate $p\left(\boldsymbol{x}_{\boldsymbol{k}} \mid z_{1: k}\right)$. The actual estimate of the state can then be taken as $E\left[p\left(\boldsymbol{x}_{\boldsymbol{k}} \mid z_{1: \boldsymbol{k}}\right)\right]$ [7].

\section{A. Observation and Transition Models}

The observational model (equation 2) is a linear function which relates the position of 24 points on the anterior portion of the external surface of the torso to the hidden state which 
describes the organ deformations. The error of the observation model represents the intrinsic error of e.g. a stereo camera system used to record external motion. $\boldsymbol{w}_{\boldsymbol{k}} \sim \mathcal{N}(0, R)$.

$$
z_{k}=B x_{k}+w_{k}
$$

The hidden state variables describe the organ deformations. The transition model describes the evolution of the organ deformations during respiration. A second order $m$ - variate vector autoregressive model $\operatorname{VAR}(2)$ is chosen which can model phenomena which exhibit regular and irregular pseudo oscillatory behaviour [8]. Uncertainty in the model is given by $\varepsilon_{\boldsymbol{k}} \sim \mathcal{N}(0, C)$.

$$
x_{k+1}=\sum_{l=1}^{p} A_{l} x_{k+1-l}+\varepsilon_{k}
$$

\section{B. The Kalman and Particle Filters}

Kalman and particle filtering frameworks represent alternative implementations of the Bayes' filtering approach, differing in the method used to represent the probability density function (pdf). The PF approximates the pdf by a weighted set of samples $w_{k}^{i}$ allowing an approximate solution to systems with complex models and non-Gaussian noise (equation 4). Further details on particle filtering can be found in [9] and for specific implementation applied to respiratory motion correction in nuclear medicine refer to [6].

$$
p\left(x_{\boldsymbol{k}} \mid z_{1: \boldsymbol{k}-\mathbf{1}}\right) \simeq \sum_{i=1}^{N} w_{k}^{i} \delta\left(\boldsymbol{x}_{\boldsymbol{k}}-\boldsymbol{x}_{\boldsymbol{k}}{ }^{i}\right)
$$

In a system described as linear with Gaussian noise, a closed form solution exists to approximate the posterior distribution; known as the Kalman filter.

$$
p\left(x_{k} \mid z_{1: k}\right) \simeq A_{k-1} \hat{x}_{k-1}-K_{k}\left(z_{k}-B A_{k-1} \hat{x}_{k-1}\right)
$$

Where $A$ and $B$ represent the transition and observation models respectively; $\hat{\boldsymbol{x}}$ represents an estimate of the hidden state. The difference between the measurement vector and the prediction of that measurement vector is termed the innovation $\boldsymbol{e}_{\boldsymbol{k}}$ (equation 6). The predicted hidden state $A_{k-1} \hat{x}_{\boldsymbol{k}-\mathbf{1}}$ is corrected optimally by the innovation; pre-multiplied by a weighting matrix called the Kalman gain $K$. The Kalman gain may be considered as the ratio of the covariance matrices of the prediction and the innovation.

$$
e_{k}=z_{k}-B A \hat{x}_{k-1}
$$

Further details on Kalman filtering can be found in [10].

\section{IMPLEMENTATION}

Model parameters were determined from a single training cycle via least squares regression utilizing the step wise least square algorithm for the autoregressive model [11]. The hidden state space was augmented to include two consecutive time indices. This allows the transition to be re-cast as a first order Markov process with state and noise vectors described by equation 7 and 8 respectively.

$$
\begin{array}{rlrl}
\widetilde{x}_{k+1} & =\left(\begin{array}{c}
x_{k+1} \\
x_{k}
\end{array}\right) \epsilon R^{m p} & \widetilde{x}_{k}=\left(\begin{array}{c}
x_{k} \\
x_{k-1}
\end{array}\right) \epsilon R^{m p} \\
\widetilde{\varepsilon}_{\boldsymbol{k}} & =\left(\begin{array}{c}
\varepsilon_{\boldsymbol{k}} \\
0
\end{array}\right) \epsilon R^{m p} & \widetilde{C} & =\left\langle\widetilde{\varepsilon}_{\boldsymbol{k}} \widetilde{\varepsilon}_{\boldsymbol{k}}^{T}\right\rangle
\end{array}
$$

The augmented state coefficient matrix and noise covariance matrix are thus described by equation 9

$$
\widetilde{A}=\left(\begin{array}{cc}
A_{1} & A_{2} \\
I & 0
\end{array}\right) \epsilon R^{m p \times m p} \quad \widetilde{C}=\left(\begin{array}{cc}
C & 0 \\
0 & 0
\end{array}\right)
$$

The observational model thus becomes (equations 10 .

$$
z_{k}=B\left(\begin{array}{c}
x_{k} \\
0
\end{array}\right)+B_{0}+w_{k}
$$

Whilst the transition model is simplified to the VAR[1] described by equation (11).

$$
\widetilde{x}_{k}=\widetilde{A} \widetilde{x}_{k-1}+\widetilde{\varepsilon}_{k}
$$

\section{A. Adaptive Transition Model and Model Implementation}

The autoregressive system matrix $\widetilde{A}$ describes the time evolution of the organ deformations. $\widetilde{A}$ is not symmetric and thus has complex eigenvectors and eigenvalues. The $m p$ eigenvalues of the system describe the modes of behaviour within the model. The argument $\theta$ of the complex components of the eigenvalues describe oscillatory behaviour of the stochastically forced oscillator.

$$
\lambda=|\lambda| \cdot e^{i \theta}
$$

The period of the pseudo sinusoidal behaviour of the hidden state $\widetilde{x}_{k}$ increases as the amplitudes of $\theta$ decreases. The autocorrelation of a chest observation for a respiratory cycle is used to estimate respiratory period. The transition model is adapted by manipulating the eigenvalues to match the period of chest observations for subsequent tests that differ to the training cycle. This allows the transition model to more accurately reflect the dynamics of the hidden state.

Performing principal component analysis (PCA) on the hidden and observable states from a small number of training data samples allows one to generate an eigenspace for future candidate hidden states and measured observed signals. Together with the mapping between the observed state and hidden state; successive estimates of the organ deformations 
can be made via tracking with the Kalman and Particle filters in PCA space. The state is also augmented in PCA space. As PCA can be considered as an orthogonal projection the actual estimates of the state can be obtained from the transpose of the projection operator.

\section{B. Testing with XCAT phantom}

To test the methodology the XCAT phantom [12] was employed which is a virtual test object allowing the user to control respiratory motion; it also provides ground truth voxel position; the phantom parameters are described in table I

\begin{tabular}{|c|c|c|}
\hline \hline Parameter & & Value \\
\hline Body & Height & $192 \mathrm{~cm}$ \\
\cline { 2 - 3 } & Weight & $95.05 \mathrm{~kg}$ \\
\hline \multirow{2}{*}{ Digitization } & Voxel size & $(3.25 \mathrm{~mm})^{3}$ \\
\cline { 2 - 3 } & Frame rate & $2 \mathrm{~s}^{-1}$ \\
\hline \hline
\end{tabular}

TABLE I

DEFAULT XCAT MALE PARAMETERS

A single training respiratory cycle was used to learn model parameters. This may be considered analogous to a multiframe low dose CT study. In this framework the hidden states $\tilde{x}_{k}$ are affine registration parameters for the internal organs for registration back to a baseline configuration $\theta$. Registration parameters are calculated at each incremental frame of the training respiratory cycle using Iterative Closest Point (ICP) [13]. The observed state, $z_{k}$, is described by the position of 24 points of the anterior portion of the external surface of the torso. Given the same training data, a test observational sequence was applied to the Kalman and Particle filters to estimate the hidden registration parameters allowing for adaptive correction for previously unseen motion.

The respiration parameters used for the training and test data are described in table II] wherein respiration is assumed to start at the end of expiration. When test data has a different cycle period to the training the transition model is adapted as described in $A$.

The XCAT voxel vector output allows one to determine the exact position of each voxel in the simulated data giving a ground truth of position for each voxel. Applying the calculated and estimated registration parameters to the motion corrupted data, coupled with the XCAT vector output, allows a comparison of the voxel positional error after motion correction has been applied (figure 2 and 3 ). In the clinical setting the estimated registration parameters would be used to correct for motion in the NM image.

\section{Simulated Emission Study}

To visualize the effect of the respiratory motion estimation framework a typical whole body PET study was simulated. It was assumed that the patients breathing pattern was limited to the seven respiratory cycles used in the experiment; with each respiratory cycle being equally probable. Five lesions of $16.25 \mathrm{~mm}$ were inserted into virtual patients lungs. Activity maps were generated based upon the standard uptake values of normal $[14]-[18]$ and diseased tissue $[19]$ reported in the
TABLE II

RESPIRATORY MOTION PARAMETERS

Motion Amplitude (cm)

\begin{tabular}{c|ccc}
\hline Dataset & Diaphragm(SI) & Chest(AP) & Cycle Period(s) \\
\hline Training & 2 & 1.2 & 5 \\
Test 1 & 1 & 0.6 & 5 \\
Test 2 & 3 & 1.8 & 5 \\
Test 3 & 4 & 2.4 & 5 \\
Test 4 & 1 & 0.6 & 3 \\
Test 5 & 1 & 1.2 & 3 \\
Test 6 & 3 & 1.8 & 3 \\
\hline
\end{tabular}

literature. Activity maps were subsequently corrected with motion estimates from the Kalman Filter. Uncorrected and corrected sinograms were simulated using ASIM [20] with a whole body count of 700 million trues and randoms. Images were reconstructed using the open source STIR [21] package.

\section{REsults}

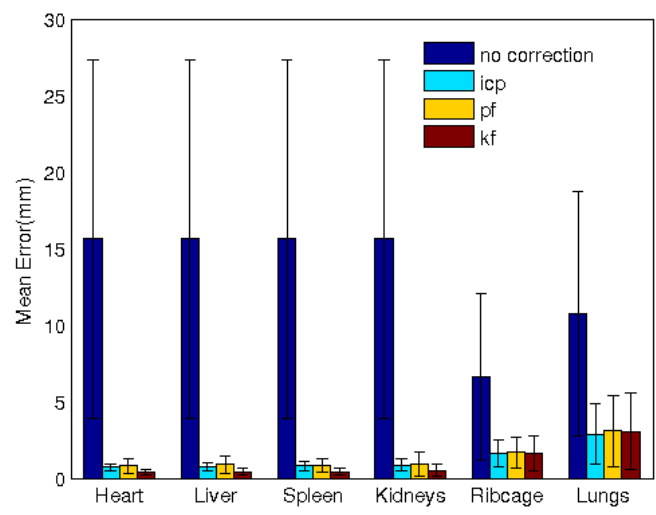

Fig. 2. Motion corrected voxel error with registration parameters calculated using ICP and estimated with the KF and PF in comparison to XCAT voxel vector $\mathrm{o} / \mathrm{p}$, averaged over all test data and all frames

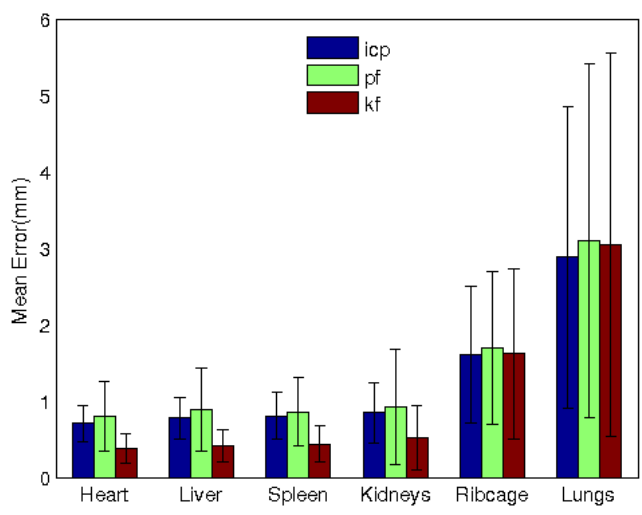

Fig. 3. Motion corrected voxel error when correction is performed with registration parameters estimated from the Kalman Filter (KF), Particle Filter (PF) and calculated from ICP

Results shown in figures 2 and figures 3 suggests the described framework offers an effective method to correct 
for unseen motion. The errors that are present are consistent with those intrinsic to using ICP as the registration method, suggesting that further error reduction is possible via development of the registration stage. Error at worse is of the order of voxel resolution $\approx 3 \mathrm{~mm}$. Utilizing the $\mathrm{KF}$ results in a marginal improvement in error in comparison to the PF. The largest mean error disparity for a given organ between the two techniques is of the order of $0.1 \mathrm{~mm}$.

The simulated emission study provides a visual assessment of the effectiveness of the proposed approach (figure 4).

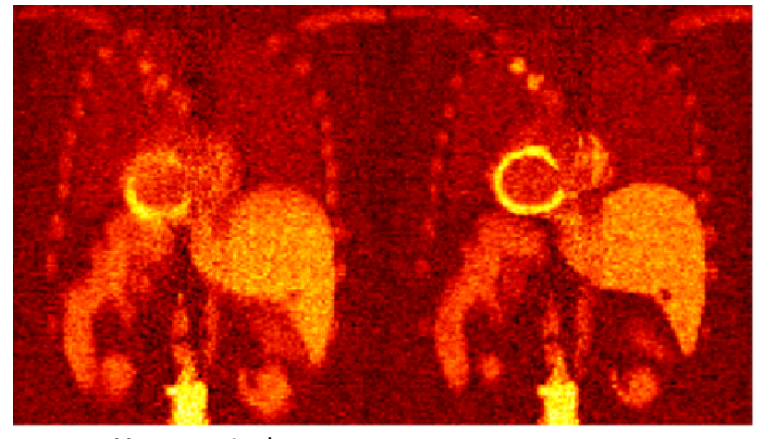

Uncorrected

Corrected

Fig. 4. Uncorrected vs. KF corrected simulated emission study

The Bayesian framework has demonstrated to improve lesion detectability and quantitative and qualitative accuracy of nuclear medicine images.

\section{CONCLUSION \& Future WORK}

The Bayesian framework offers an effective method to correct for previously unseen motion. Little significant errors are introduced over and above that which is intrinsic to ICP as organ wise registration method. The residual error is at worse of the order of voxel resolution $\approx 3 \mathrm{~mm}$. The adaptive transition model accounts for respiratory cycles of different period to that of the training data. Future work will test the framework on clinical data and investigate the dynamical characteristics of respiratory motion.

\section{REFERENCES}

[1] S. A. Nehmeh and Y. E. Erdi, "Respiratory Motion in Positron Emission Tomography/Computed Tomography: A Review," Semin Nucl Med, vol. 38, no. 3, pp. 167-176, 2008.

[2] C. Liu, A. M. Alessio, and P. E. Kinahan, "Respiratory motion correction for quantitative PET/CT using all detected events with internalexternal motion correlation," Medical Physics, vol. 38, no. 5, p. 2715, 2011. [Online]. Available: http://link.aip.org/link/MPHYA6/v38/ i5/p2715/s1\&Agg=doi

[3] W. Bai and S. M. Brady, "Motion Correction and Attenuation Correction in Thoracic PET Imaging," Bone, no. x, pp. 3593-3598, 2010.

[4] A. P. King, C. Buerger, C. Tsoumpas, P. K. Marsden, and T. Schaeffter, "Thoracic respiratory motion estimation from MRI using a statistical model and a 2-D image navigator," Med. Image Anal., vol. 16, no. 1, pp. 252-264, 2012.

[5] K. Wells, B. Goswami, A. A. A. Rahni, J. Jones, M. Alnowami, E. Lewis, and M. Guy, "A Flexible Approach to Motion Correction in Nuclear Medicine," in IEEE NSS/MIC Conf Rec, Nov. 2009, pp. 2534 2539.

[6] A. A. A. Rahni, K. Wells, E. Lewis, and Others, "An Iterative Particle Filter Approach for Respiratory Motion Estimation in Nuclear Medicine Imaging," IEEE.Trans.Med.Imag., vol. 7962, p. 79624C, 2011.
[7] B. Ristic, S. Arulampalam, and N. Gordon, Eds., Beyond the Kalman Filter: Particle Filters for Tracking Applications. Artech House, 2004.

[8] C. M. Stralkowski, S. M. Wu, and R. E. DeVon, "Charts for the Interpretation and Estimation of the Second Order Autoregressive Model," Amer. Soc. for Quality, vol. 12, no. 3, pp. 669-685, 1970.

[9] B. Ristic, S. Arulampalam, and N. Gordon, Beyond the Kalman Filter: Particle Filters for Tracking Applications. Artech House, Jan. 2004, vol. 103. [Online]. Available: http://www.ncbi.nlm.nih.gov/pubmed/ 23135598

[10] B. D. O. Anderson and J. B. Moore, Optimal Filtering. Prentice-Hakk, 1979.

[11] A. Neumaier and T. Schneider, "Estimation of Parameters and Eigenmodes of Multivariate Autoregressive Models," ACM Transactions on Mathematical Software, vol. 27, no. 1, pp. 27-57, Mar. 2001.

[12] W. P. Segars, G. Sturgeon, S. Mendonca, and Others, "4D XCAT Phantom for Multimodality Imaging Research,” J.Med.Phys., vol. 37, no. 9, pp. 4902-4915, 2010.

[13] J. Jones, E. Lewis, M. Guy, and Others, "A Virtual Dissection Based Registration to Model Patient-Specific Respiratory Motion," in IEEE.Med.Imag.Conf., 2009, pp. 3571-3576.

[14] R. Minamimoto, N. Takahashi, and T. Inoue, "FDG-PET of Patients with Suspected Renal Failure: Standardized Uptake Values in Normal Tissues." Annals of Nucl.Med., vol. 21, no. 4, pp. 217-22, Jun. 2007.

[15] A. Rominger, T. Saam, S. Wolpers, and Others, "18F-FDG PET/CT Identifies Patients at Risk for Future Vascular Events in an Otherwise Asymptomatic Cohort With Neoplastic Disease." J.Nucl.Med., vol. 50, no. 10 , pp. 1611-1620, Oct. 2009.

[16] S. Zincirkeser, E. Sahin, and M. Halac, "Standardized Uptake Values of Normal Organs on 18 F-Fluorodeoxyglucose Positron Emission Tomography and Computed Tomography Imaging," The Journal of International Medical Research, vol. 35, pp. 231-236, 2007.

[17] Y. Tayamaa, N. Takahashi, T. Oka, and Others, "Clinical evaluation of the effect of attenuation correction technique on 18F-fluoride PET images." Annals of Nucl.Med., vol. 21, no. 2, pp. 93-99, Feb. 2007.

[18] A. Chung, D. Liou, S. Karlan, and Others, "Preoperative FDG-PET for Axillary Metastases in Patients with Breast Cancer." Archives of surgery (Chicago, Ill. : 1960), vol. 141, no. 8, pp. 783-8; discussion 788-9, Aug. 2006.

[19] I. C. Okereke, S. P. Gangadharan, and M. S. Kent, "Standard Uptake Value Predicts Survival in Non-Small Cell Lung Cancer." The Annals of Thoracic Surgery, vol. 88, no. 3, pp. 911-5; discussion 915-6, Sep. 2009.

[20] C. Corntat, P. E. Kinahan, M. Defrise, C. Miche, C. Lartizien, and D. W. T. S. Member, "Simulating Whole-Body PET Scanning with Rapid Analytical Methods," Simulation, vol. 00, no. c, pp. 1260-1264, 2000.

[21] K. Thielemans, C. Tsoumpas, S. Mustafovic, T. Beisel, P. Aguiar, N. Dikaios, and M. W. Jacobson, "STIR: software for tomographic image reconstruction release 2." Physics in medicine and biology, vol. 57, no. 4, pp. 867-83, Feb. 2012. [Online]. Available: http://www.ncbi.nlm.nih.gov/pubmed/22290410 